\title{
COMPOSIÇÃO CENTESIMAL E DE MINERAIS EM CASCAS DE FRUTAS
}

\author{
Jussara A. Melo GONDIM ${ }^{2, *}$, Maria de Fátima V. MOURA², Aécia S. DANTAS ${ }^{3}$, \\ Rina Lourena S. MEDEIROS ${ }^{3}$, Klécia M. SANTOS ${ }^{4}$
}

\begin{abstract}
RESUMO
Informações sobre a composição de alimentos de origem agrícola cultivados em solos brasileiros são escassas, e mais ainda de alimentos provenientes do Nordeste. O desconhecimento dos princípios nutritivos dos alimentos induz ao mau aproveitamento, o que ocasiona o desperdício de toneladas de recursos alimentares. Com o objetivo de incentivar o reaproveitamento de alimentos e oferecer uma alternativa nutritiva de dieta a baixo custo, foram analisadas as cascas de algumas frutas que normalmente são desprezadas. No presente trabalho, foi determinada a composição centesimal de 7 elementos minerais com importância nutricional ( $\mathrm{Ca}, \mathrm{Cu}, \mathrm{Fe}, \mathrm{K}, \mathrm{Mg}, \mathrm{Na}, \mathrm{Zn}$ ) em 7 tipos diferentes de cascas de frutas: abacate, abacaxi, banana, mamão, maracujá, melão e tangerina, cultivadas no Estado do Rio Grande do Norte. As análises químicas mostraram que as cascas das frutas apresentam, em geral, teores de nutrientes maiores do que os das suas respectivas partes comestíveis, conforme verificado na literatura. Desta forma, pode-se considerar que as cascas das frutas analisadas podem ser úteis como fontes alternativas de alimento ou como ingredientes para obtenção de preparações processadas.

Palavras-chave: composição centesimal, minerais, cascas de frutas.
\end{abstract}

\section{SUMMARY}

CENTESIMAL COMPOSITION AND MINERALS IN PEELS OF FRUITS. Information on the composition of Brazilian foods is scant, mainly of Northeastern origin. Still, the ignorance of the nutritious principles of the foods, as well as improper use, cause the waste of tons of alimentary resources. With the objective of motivating the reuse of foods and offering a nutritious alternative of diet at a low cost, the peels of some fruits were analyzed, which are usually discarded. In the present work, it was determined the centesimal composition and 7 mineral elements with nutritious importance ( $\mathrm{Ca}, \mathrm{Cu}, \mathrm{Fe}, \mathrm{K}, \mathrm{Mg}, \mathrm{Na}, \mathrm{Zn})$ in 7 different types from peels of fruits: avocado, pineapple, banana, papaya, passion fruit, melon and tangerine, cultivated in Rio Grande do Norte State. The chemical analyses showed that the peels of the fruits present, in general, larger content of nutrients than the respective edible parts verified in the literature. This way, it can be considered that the peels of the analyzed fruits can be used as alternative source of food or as ingredients as to obtain processed preparations.

Keywords: centesimal composition, minerals, peels of fruits.

\section{1 - INTRODUÇÃO}

A Ingestão Diária Recomendada (IDR) é a quantidade de vitaminas, minerais e proteínas que deve ser consumida diariamente para atender às necessidades nutricionais da maior parte dos indivíduos e grupos de pessoas de uma população sadia [2].

Para a população consumir equilibradamente os nutrientes de acordo com a IDR, são necessários dados sobre composições de alimentos. Essas composições são importantes para inúmeras atividades, como para avaliar o suprimento e o consumo alimentar de um país, verificar a adequação nutricional da dieta de indivíduos e de populações, avaliar o estado nutricional, desenvolver pesquisas sobre as relações entre dieta e doença, em planejamento agropecuário, na indústria de alimentos, além de outras [8].

\footnotetext{
${ }^{1}$ Recebido para publicação em 20/05/2005. Aceito para publicação em 04/10/2005 (001533).

${ }^{2}$ Departamento de Farmácia.

${ }^{3}$ Departamento de Química - Laboratório de Química Analítica.

${ }^{4}$ Universidade Federal do Rio Grande do Norte - UFRN, Caixa Postal 1524

- Lagoa Nova. CEP: 59072-970, Natal-RN.E-mail: jussara_gondim@ bol.com.br.

"A quem a correspondência deve ser enviada.
}

No entanto, devido às dificuldades econômicas atuais, torna-se cada vez mais difícil adquirir alimentos adequados ao consumo do dia-a-dia, razão pela qual a alimentação equilibrada é atualmente uma das maiores preocupações do nosso cotidiano.

A fome e o desperdício de alimentos são dois dos maiores problemas que o Brasil enfrenta, constituindose em um dos paradoxos de nosso país. Produzimos 140 milhões de toneladas de alimentos por ano, somos um dos maiores exportadores de produtos agrícolas do mundo e, ao mesmo tempo, temos milhões de excluídos, sem acesso ao alimento em quantidade e/ou qualidade.

Frutas e vegetais são exemplos de importantes fontes de elementos essenciais. Os minerais desempenham uma função vital no peculiar desenvolvimento e boa saúde do corpo humano e as frutas são consideradas as principais fontes de minerais necessários na dieta humana [4].

Como o homem necessita, de qualquer modo, de uma alimentação sadia, rica em nutrientes, isto pode ser alcançado com partes de alimentos que normalmente são desprezadas. Sendo assim, é importante a utilização de cascas, talos e folhas, pois o aproveitamento integral dos alimentos, além de diminuir os gastos com alimentação e melhorar a 
qualidade nutricional do cardápio, reduz o desperdício de alimentos e torna possível a criação de novas receitas, como, por exemplo, sucos, doces, geléias e farinhas.

Com o objetivo de incentivar o reaproveitamento de alimentos e oferecer uma alternativa nutritiva de dieta a baixo custo, foram analisadas as cascas de algumas frutas que normalmente são desprezadas. Para isso, foram determinados a composição centesimal e 7 elementos minerais com importância nutricional (Ca, Cu, Fe, K, Mg, Na, Zn) em 7 tipos diferentes de cascas de frutas: abacate, abacaxi, banana, mamão, maracujá, melão e tangerina cultivados no Estado do Rio Grande do Norte.

\section{2 - MATERIAL E MÉTODOS}

\section{1 - Amostras}

Foram analisados 7 tipos diferentes de cascas de frutas maduras: abacate (Persea americana), abacaxi (Ananas comosus), banana (Musa sp.), mamão (Carica papaya), maracujá (Passiflora edulis), melão (Cucumis melo) e tangerina (Citrus reticulata), cultivados no Estado do Rio Grande do Norte.

As cascas das frutas foram lavadas em água corrente e separadas das respectivas polpas. Logo após, uma a uma, foram homogeneizadas em um multiprocessador para a realização das análises de umidade. O restante foi colocado em uma estufa ventilada $\left(60^{\circ} \mathrm{C}\right)$ para a secagem e execução das outras análises.

\section{2 - Análise da composição centesimal}

As determinações de umidade residual $\left(105^{\circ} \mathrm{C}\right)$, proteínas, lipídeos e cinzas foram realizadas em triplicatas de amostras, segundo métodos da AOAC [1]. A fração Nifext foi obtida pelo cálculo da diferença das outras frações analisadas. $\mathrm{O}$ valor calórico total foi calculado a partir da soma das calorias correspondentes para proteínas, lipídeos e carboidratos (Nifext). Os resultados foram expressos de forma integral.

\section{3 - Análise dos teores de elementos minerais}

Para as determinações de cálcio, cobre, ferro, sódio, potássio, magnésio e zinco, foi utilizado um espectrômetro de absorção atômica, modelo SpectrAA 110, Varian, calibrado em condições específicas de comprimento de onda, fenda e mistura dos gases para cada elemento.

Para a construção das curvas de calibração, foram utilizadas ampolas de padrões para absorção atômica Merck devidamente diluídas com água deionizada. As análises foram realizadas em triplicata.

\section{3 - RESULTADOS E DISCUSSÃO}

Os resultados da composição centesimal e de minerais das cascas de frutas cultivadas no Estado do Rio Grande do Norte encontram-se na Tabela 1.

A amostra da casca de melão teve o maior teor de umidade, 93,23 g; em compensação, apresentou o menor valor calórico e de minerais. A amostra da casca de abacate teve o maior teor de lipídeos, 11,04 g, havendo uma diferença muito grande em relação às outras amostras, as quais não passaram de 1,0 g. A amostra da casca de tangerina revelou os maiores teores para o restante dos outros parâmetros, provavelmente devido à menor taxa de umidade, 49,10 g.

Dentre os elementos minerais analisados, o que apresentou maiores concentrações foi o potássio, seguido do cálcio e magnésio. O que apresentou menor teor foi o cobre, seguido do zinco.

Com estes resultados, foi possível calcular as contribuições percentuais das amostras de cascas de frutas analisadas em relação à Ingestão Diária Recomendada (IDR) para cada nutriente [2 e 5]. Estes dados, considerando-se os requerimentos nutricionais de um adulto, encontramse na Tabela 2.

A amostra da casca de abacate pode ser considerada uma boa fonte de lipídeos, pois fornece $14 \%$ da IDR. As outras cascas são pobres em relação a esse nutriente, podendo

TABELA 1 - Resultado do teor de nutrientes das cascas de frutas analisadas

\begin{tabular}{|c|c|c|c|c|c|c|c|}
\hline \multirow{2}{*}{ Parâmetro } & \multicolumn{7}{|c|}{$100 \mathrm{~g}$ de amostra in natura das cascas de frutas } \\
\hline & Abacate & Abacaxi & Banana & Mamão & Maracujá & Melão & Tangerina \\
\hline Umidade (g) & 76,95 & 78,13 & 89,47 & 90,63 & 87,64 & 93,23 & 49,10 \\
\hline Cinzas (g) & 0,75 & 1,03 & 0,95 & 0,82 & 0,57 & 0,96 & 1,75 \\
\hline Lipídeos (g) & 11,04 & 0,55 & 0,99 & 0,08 & 0,01 & 0,10 & 0,64 \\
\hline Proteínas (g) & 1,51 & 1,45 & 1,69 & 1,56 & 0,67 & 1,24 & 2,49 \\
\hline Fibras (g) & 6,85 & 3,89 & 1,99 & 1,20 & 4,33 & 1,42 & 10,38 \\
\hline Carboidratos (g) & 2,90 & 14,95 & 4,91 & 5,71 & 6,78 & 3,05 & 35,64 \\
\hline Calorias (Kcal) & 117,02 & 70,55 & 35,30 & 29,80 & 29,91 & 18,05 & 158,30 \\
\hline Cálcio (mg) & 123,94 & 76,44 & 66,71 & 55,41 & 44,51 & 14,69 & 478,98 \\
\hline Ferro (mg) & 2,18 & 0,71 & 1,26 & 1,10 & 0,89 & 0,40 & 4,77 \\
\hline Sódio (mg) & 76,75 & 62,63 & 54,27 & 53,24 & 43,77 & 8,54 & 77,76 \\
\hline Magnésio (mg) & 26,24 & 26,79 & 29,96 & 24,52 & 27,82 & 13,27 & 159,59 \\
\hline Zinco (mg) & 1,24 & 0,45 & 1,00 & 0,56 & 0,32 & 0,23 & 2,83 \\
\hline Cobre (mg) & 0,18 & 0,11 & 0,10 & 0,11 & 0,04 & 0,07 & 0,58 \\
\hline Potássio (mg) & 236,70 & 285,87 & 300,92 & 263,52 & 178,40 & 110,39 & 598,36 \\
\hline
\end{tabular}


TABELA 2 - Percentual da Ingestão Diária Recomendada (IDR) para um adulto

\begin{tabular}{|c|c|c|c|c|c|c|c|}
\hline \multirow{2}{*}{ Parâmetro } & \multicolumn{7}{|c|}{ \% IDR em relação a $100 \mathrm{~g}$ de amostra in natura das cascas de frutas } \\
\hline & Abacate & Abacaxi & Banana & Mamão & Maracujá & Melão & Tangerina \\
\hline Lipídeos & 14 & 1 & 1 & 0 & 0 & 0 & 1 \\
\hline Proteínas & 3 & 3 & 3 & 3 & 1 & 2 & 5 \\
\hline Fibras & 23 & 13 & 7 & 4 & 14 & 5 & 35 \\
\hline Carboidratos & 1 & 4 & 1 & 1 & 1 & 1 & 6 \\
\hline Calorias & 5 & 3 & 1 & 1 & 1 & 1 & 6 \\
\hline Cálcio & 15 & 9 & 8 & 7 & 5 & 2 & 60 \\
\hline Ferro & 14 & 5 & 8 & 7 & 6 & 3 & 32 \\
\hline Sódio & 3 & 3 & 2 & 2 & 2 & 0 & 4 \\
\hline Magnésio & 9 & 9 & 10 & 8 & 9 & 4 & 53 \\
\hline Zinco & 8 & 3 & 7 & 4 & 2 & 1 & 19 \\
\hline Cobre & 6 & 4 & 6 & 4 & 1 & 2 & 19 \\
\hline Potássio & 12 & 14 & 15 & 13 & 9 & 5 & 30 \\
\hline
\end{tabular}

TABELA 3 - Teor de nutrientes das partes comestíveis das frutas

\begin{tabular}{|c|c|c|c|c|c|c|c|}
\hline \multirow{2}{*}{ Parâmetro } & \multicolumn{7}{|c|}{$100 \mathrm{~g}$ de amostra in natura das partes comestíveis das frutas } \\
\hline & Abacate & Abacaxi & Banana & Mamão & Maracujá & Melão & Tangerina \\
\hline Cinzas (g) & 0,5 & 0,4 & 0,8 & 0,6 & 0,8 & 0,5 & 0,6 \\
\hline Proteínas (g) & 1 & 1 & 1 & 1 & 2 & 1 & 1 \\
\hline Fibras (g) & 6,3 & 1 & 1,5 & 1,8 & 1,1 & 0,3 & 0,9 \\
\hline Carboidratos (g) & 6 & 12 & 34 & 12 & 12 & 8 & 10 \\
\hline Calorias (Kcal) & 96 & 48 & 128 & 45 & 68 & 29 & 38 \\
\hline Sódio (mg) & $<0,4$ & $<0,4$ & $<0,4$ & 3 & 2 & 11 & $<0,4$ \\
\hline Magnésio (mg) & 15 & 18 & 24 & 17 & 28 & 6 & 8 \\
\hline Zinco (mg) & 0,2 & 0,3 & 0,3 & 0,2 & 0,6 & 0,2 & 0,1 \\
\hline Cobre (mg) & 0,15 & 0,11 & 0,05 & 1,36 & 0,19 & 0,04 & 0,03 \\
\hline Potássio (mg) & 206 & 131 & 328 & 222 & 338 & 216 & 131 \\
\hline
\end{tabular}

Fonte: Guilherme Franco - Tabela de Composição Química dos Alimentos - Editora Atheneu

ser utilizadas em dietas de redução de peso. Para todos os outros parâmetros, a amostra da casca de tangerina revelou os maiores percentuais. Em relação às fibras, podemos considerar as cascas de tangerina, abacate, maracujá e abacaxi como boas fontes, regularizando as funções intestinais.

Os resultados das análises verificados na Tabela 1 podem ser comparados com os dos teores de nutrientes das partes comestíveis das frutas constantes na Tabela 3 verificados na literatura [3].

Comparando a Tabela 1 com a Tabela 3, podemos verificar que as cascas analisadas apresentam quantidades de nutrientes maiores que as suas respectivas partes comestíveis. O teor de lipídeos é o maior na polpa do abacate, como acontece na sua casca. A polpa do melão também apresenta menor umidade e valor calórico. No entanto, a polpa da tangerina não apresenta os maiores teores em nenhum dos parâmetros.

\section{4 - CONCLUSÕES}

As análises químicas mostraram que as cascas das frutas apresentam, em geral, teores de nutrientes maiores do que os das suas respectivas partes comestíveis. Desta forma, as cascas das frutas analisadas podem ser consideradas como fonte alternativa de nutrientes, evitando o desperdício de alimentos. A próxima etapa deste trabalho será a pesquisa de fatores anti-nutricionais nas amostras em questão.

\section{5 - REFERÊNCIAS BIBLIOGRÁFICAS}

[1] Association of Official Analytical Chemists. Official Methods of Analysis. Arlington, AOAC, 1984.

[2] BRASIL. Portaria MS no 33, de 13 de janeiro de 1998. Ingestão Diária Recomendada (IDR) para proteínas, vitaminas e minerais. Diário Oficial da República Federativa do Brasil, Brasília, 16 de janeiro de 1998.

[3] FRANCO, G.V.E. Nutrição. Texto Básico e Tabela de Composição Química de Alimentos. $6^{\mathrm{a}}$ ed. São Paulo. Livraria Atheneu, 1982.

[4] HARDISSON, A. et al. Mineral composition of the banana (Musa acuminata) from the island of Tenerife. Food Chemistry, 2001, vol. 73, p. 153-161.

[5] National Research Council. Recommended Dietary Allowances. National Academy Press, Washington DC, 1989.

[6] PEDROSA, L.F.C. \& COZZOLINO, S.M.F. Composição centesimal e de minerais de mariscos crus e cozidos da cidade de Natal/RN. Ciênc. Tecnol. Aliment., maio/ago. 2001, vol. 21, n. 2, p. 154-157.

[7] SOARES, L.M.V.; SHISHIDO, K.; MORAES, A.M.M. et al. Composição mineral de sucos concentrados de frutas brasileiras. Ciênc. Tecnol. Aliment., abr./jun. 2004, vol. 24, n. 2, p. 202-206.

[8] TORRES, E.A.F.S; CAMPOS, N.C.; DUARTE, M. et al. Composição centesimal e valor calórico de alimentos de origem animal. Ciênc. Tecnol. Aliment., maio/ago. 2000, vol. 20, n. 2, p. 145-150. 